\title{
INTERPOLATION SERIES IN LOCAL FIELDS OF PRIME CHARACTERISTIC
}

\author{
CARL G. WAGNER
}

1. Introduction. In 1944 Dieudonne [3] proved a $p$-adic analogue of the Weierstrass Approximation Theorem by an inductive argument involving the polynomial approximation of certain continuous characteristic functions. In 1958 Mahler [4] proved the sharper result that each continuous $p$-adic function $f$ defined on the $p$-adic integers is the uniform limit of the "interpolation series"

$$
f(t)=\sum_{n=0}^{\infty} \Delta^{n} f(0)\left(\begin{array}{l}
t \\
n
\end{array}\right)
$$

where

$$
\Delta^{n} f(0)=\sum_{k=0}^{n}(-1)^{k}\left(\begin{array}{l}
n \\
k
\end{array}\right) f(n-k) .
$$

The crucial step in Mahler's proof involves showing that $\lim _{n \rightarrow \infty} \Delta^{n} f(0)=0$ for the $p$-adic topology, and he demonstrates this by passing to a certain cyclotomic extension of the rationals. In fact, this follows quickly from Dieudonne's theorem for if $p(t)$ is a polynomial of degree $r$ for which $|f(t)-p(t)|_{p}<\epsilon$ for $t \varepsilon Z_{p}$, then $\left|\Delta^{n} f(0)-\Delta^{n} p(0)\right|_{p}<\epsilon$ for all $n$. Hence if $n>r, \Delta^{n} p(0)=0$ and $\left|\Delta^{n} f(0)\right|_{\nu}<\epsilon$.

In the present paper we use the above idea to simplify our earlier proof of a Mahler type theorem for continuous functions on the ring $V$ of formal power series over a finite field $G F(q)$ [5]. Although the proof by Dieudonné admits a straightforward generalization to any locally compact non-archimedean field, in this case we accomplish the polynomial approximation of the relevant characteristic functions without recourse to induction by using certain powers of the Carlitz polynomials $G_{a^{r}-1}^{\prime}(t) / g_{a^{r-1}}$ [1]. We conclude by giving a sufficient condition for the differentiability of a function $f$ defined on $V$.

2. Preliminaries. Let $G F[q, x]$ be the ring of polynomials over the finite field $G F(q)$ of characteristic $p$ and let $G F(q, x)$ be the quotient field of $G F[q, x]$. Denote by $V$ the ring of formal power series over $G F(q)$ and by $F$ the field of formal power series over $G F(q)$. Set $|0|=0$. If $\alpha \varepsilon F-\{0\}$ is given by

$$
\alpha=\sum_{i=-\infty}^{\infty} a_{i} x^{i}
$$

Received December 13, 1971. This research was supported in part by the University of Tennessee Faculty Research Fund. 
where $a_{i} \varepsilon G F(q)$ and all but a finite number of the $a_{i}$ 's vanish for $i<0$, then set $v(\alpha)=k$ and

$$
|\alpha|=b^{o(\alpha)}
$$

where $0<b<1$ and $k$ is the smallest subscript $i$ in (2.1) for which $a_{i} \neq 0$. Then || is a discrete, non-archimedean absolute value on $F$ and $F$ is complete with respect to this absolute value. Obviously $G F[q, x]$ is dense in $V$ as is $G F(q, x)$ in $F$. The valuation ring of $F$ is $V$, and $V$ is compact and open in $F$ [5; 392]. Also, addition and multiplication are continuous operations in $F$ so that polynomials over $F$ define continuous functions.

Following Carlitz we define a sequence of polynomials $\Psi_{n}(t)$ over $G F[q, x]$ by

$$
\Psi_{n}(t)=\prod_{\text {deg } m<n}(t-m),
$$

where the above product extends over all $m \varepsilon G F[q, x]$ of degree less than $n$ (including 0 ). Then $[2 ; 140]$

$$
\Psi_{n}(t)=\sum_{i=0}^{n}(-1)^{n-i}\left[\begin{array}{c}
n \\
i
\end{array}\right] t^{a^{i}},
$$

where

$$
\left[\begin{array}{l}
n \\
i
\end{array}\right]=\frac{F_{n}}{F_{i} L_{n-i}^{a^{i}}}, \quad\left[\begin{array}{l}
n \\
0
\end{array}\right]=\frac{F_{n}}{L_{n}}, \quad\left[\begin{array}{l}
n \\
n
\end{array}\right]=1
$$

and

$$
\begin{aligned}
& F_{n}=[n][n-1]^{e} \cdots[1]^{a^{n-1}}, \quad F_{0}=1 \\
& L_{n}=[n][n-1] \cdots[1], \quad L_{0}=1 \\
& {[r]=x^{a^{+}}-x .}
\end{aligned}
$$

Following [1] we define polynomials $G_{n}(t)$ and $G_{n}^{\prime}(t)$ over $G F[q, x]$ and $g_{n} \varepsilon G F[q, x]$ as follows. If

$$
n=e_{0}+e_{1} q+\cdots+e_{\imath} q^{s}, \quad 0 \leq e_{i}<q,
$$

then set

$$
G_{n}(t)=\Psi_{0}^{\varepsilon_{0}}(t) \cdots \Psi_{\bullet}^{\bullet \cdot}(t)
$$

and

$$
G_{n}^{\prime}(t)=\prod_{i=0}^{s} G_{e i a^{i}}^{\prime}(t)
$$

where

$$
G_{e q^{i}}^{\prime}(t)= \begin{cases}\Psi_{i}^{e}(t) & 0 \leq e<q-1 \\ \Psi_{i}^{e}(t)-F_{i}^{e} & e=q-1\end{cases}
$$


and

$$
g_{n}=F_{1}^{e_{2}} \cdots F_{8}^{e \cdot}, \quad g_{0}=1 .
$$

We mention that $G_{n}(t) / g_{n}$ and $G_{n}^{\prime}(t) / g_{n}$ are integral valued polynomials over $G F(q, x)$, i.e., for all $m \varepsilon G F[q, x], G_{n}(m) / g_{n}, G_{n}^{\prime}(m) / g_{n} \varepsilon G F[q, x][1 ; 503]$.

If $H$ is any extension field of $G F(q, x)$, since $\operatorname{deg} G_{n}(t)=n$, it follows that $\left(G_{n}(t) / g_{n}\right)$ is an ordered basis of the $H$-vector space $H[t]$. Indeed for any $h(t) \varepsilon H[t]$ of degree $\leq n$ we have $[1 ; 491]$ the unique representation

$$
h(t)=\sum_{i=0}^{n} A_{i} \frac{G_{i}(t)}{g_{i}},
$$

where

$$
A_{i}=(-1)^{r} \sum_{\operatorname{deg} m<r} \frac{G_{0^{r}-1-i}^{\prime}(m)}{g_{a^{r}-1-i}} h(m), \quad m \varepsilon G F[q, x]
$$

and $i<q^{r}$. We emphasize that for $i>n$ Formula (2.13) yields $A_{i}=0$, so we could have written the sum in (2.12) with upper limit $\infty$. In the sequel we shall expand an arbitrary continuous function $f: V \rightarrow F$ in a (genuinely) infinite series resembling (2.12).

3. Characteristic functions. For all nonnegative integers $k$ define a function $\chi_{k}$ on $V$ by $\chi_{k}(t)=1$ if $|t| \leq b^{k}$ and $\chi_{k}(t)=0$ if $b^{k}<|t| \leq 1$. As the characteristic function of an open-closed ball about $0, \chi_{k}$ is continuous. The following theorem shows that it may be uniformly approximated by polynomials over $G F(q, x)$.

Theorem A. For $k \geq 0$ let

$$
C_{k}(t)=(-1)^{k} G_{a^{k-1}}^{\prime}(t) / g_{a^{k-1}} .
$$

Then for all $t \varepsilon V$ and for all natural numbers $s$

$$
\left|C_{k}^{p^{*}}(t)-\chi_{k}(t)\right| \leq b^{p^{*}}
$$

where $p$ is the characteristic of $F$.

Proof. By $[2 ; 141] G_{a^{k-1}}^{\prime}(t)=\Psi_{k}(t) / t$. If $|t| \leq b^{k}$, then $t=x^{k} \mu$, where $\mu \varepsilon V$. It follows from (2.4), (2.5), (2.6) and (2.11) that $C_{k}(0)=1$, and so we may assume that $\mu \neq 0$. Then by these same four formulae

$$
C_{k}\left(x^{k} \mu\right)=(-1)^{k} \frac{L_{k} \Psi_{k}\left(x^{k} \mu\right)}{F_{k} x^{k} \mu}=1+\sum_{i=1}^{k}(-1)^{2 k-i} \frac{\left(x^{k} \mu\right)^{q^{i}-1} L_{k}}{F_{i} L_{k-j}^{q^{j}}} .
$$

But each of the terms other than 1 in $(3.3)$ is congruent to zero $(\bmod x)$ for if $1 \leq j \leq k$, then

$$
\begin{aligned}
v\left(\left(x^{k} \mu\right)^{a^{i}-1} L_{k} / F_{i} L_{k-i}^{a^{i}}\right) & \geq\left(q^{i}-1\right) k+k-\left(1+q+\cdots+q^{i-1}\right)-q^{i}(k-j) \\
& =j q^{i}-\left(1+q+\cdots+q^{i-1}\right)>0 .
\end{aligned}
$$


Hence there exists a $\beta \varepsilon V$ such that

$$
C_{k}\left(x^{k} \mu\right)=1+\beta x
$$

and so for all $s \geq 1$

$$
C_{k}^{p^{*}}\left(x^{k} \mu\right)=1+(\beta x)^{p^{*}}
$$

from which (3.2) follows for $|t| \leq b^{k}$.

If $b^{k}<|t|<1$ and since $\left|\Psi_{k}(t) / F_{k}\right| \leq 1$ for all $t \varepsilon V[6 ; \S 3]$, then

$$
\left|C_{k}^{p^{*}}(t)-\chi_{k}(t)\right|=\left|C_{k}(t)\right|^{p^{*}}=\left|\frac{L_{k} \Psi_{k}(t)}{t F_{k}}\right|^{p^{*}} \leq b^{p^{*}} .
$$

Remark. It follows from (3.2) by translation that for all $\alpha \varepsilon V$

$$
\left|C_{k}^{p^{*}}(t-\alpha)-\chi_{k}(t-\alpha)\right| \leq b^{p^{*}} .
$$

Hence the characteristic function of any open-closed ball in $V$ may be uniformly approximated by polynomials.

4. Theorem B. Let $f: V \rightarrow F$ be continuous and for all $i \geq 0$ set

$$
A_{i}=(-1)^{r} \sum_{\operatorname{deg} m<r} \frac{G_{q^{r}-1-i}^{\prime}(m)}{g_{a^{r}-1-i}} f(m)
$$

where $i<q^{r}$ (any such $r$ yields the same value for $A_{i}[1 ; 492]$ ) and the sum in (4.1) extends over all $m \varepsilon G F[q, x]$ of degree $<r$. Then

$$
\sum_{i=0}^{\infty} A_{i} \frac{G_{i}(t)}{g_{i}}
$$

converges uniformly to $f(t)$ for all $t \varepsilon V$.

Proof. Since $\left|G_{i}(t) / g_{i}\right| \leq 1$ for all $t \varepsilon V[6 ; \S 3]$ and || is non-archimedean, the uniform convergence of (4.2) would follow from a proof that $\lim _{i \rightarrow \infty} A_{i}=0$. Hence, given $s \geq 0$, we seek $N=N(s)$ such that $i>N$ implies that $\left|A_{i}\right| \leq b^{s}$. Since $V$ is compact, $f$ is bounded, and we may assume with no loss of generality that $f: V \rightarrow V$. Also $f$ is uniformly continuous, and so there exists a $k=k(s)$ such that $\left|t_{1}-t_{2}\right| \leq b^{k}$ implies $\left|f\left(t_{1}\right)-f\left(t_{2}\right)\right| \leq b^{s}$ for $t_{1}, t_{2} \varepsilon V$.

For $m \varepsilon G F[q, x]$ suppose that $f(m)=\sum_{i=0}^{\infty} a_{i} x^{i}$. Set $f_{8}(m)=a_{0}+a_{1} x+$ $\cdots+a_{s-1} x^{s-1}$. This defines a function $f_{8}: G F[q, x] \rightarrow G F[q, x]$ for which

$$
\left|f_{s}(m)-f(m)\right| \leq b^{s}
$$

for all $m_{\varepsilon} G F[q, x]$. Furthermore, $f$ is periodic $\left(\bmod x^{k}\right)$ for if $m_{1} \equiv m_{2}\left(\bmod x^{k}\right)$, i.e., if $\left|m_{1}-m_{2}\right| \leq b^{k}$, then by (4.3) and the uniform continuity of $f$ it follows that $\left|f_{s}\left(m_{1}\right)-f_{s}\left(m_{2}\right)\right| \leq b^{s}$. Hence $f_{s}\left(m_{1}\right)=f_{s}\left(m_{2}\right)$ since distinct values of $f_{\text {. }}$ are incongruent $\left(\bmod x^{8}\right)$.

Corresponding to (4.1) we define a sequence $\left(B_{i}\right)$ in $G F[q, x]$ by

$$
B_{i}=(-1)^{r} \sum_{\text {deg } m<r} \frac{G_{a^{r}-1-i}^{\prime}(m)}{g_{a^{r-1-i}}} f_{8}(m),
$$


where $i<q^{r}$. Since $G_{a^{r-1-i}}^{\prime}(m) / g_{a^{r-1-i}} \varepsilon G F[q, x]$, it follows from (4.3) that for all $i \geq 0$

$$
\left|A_{i}-B_{i}\right| \leq b^{s}
$$

By (4.4) and the periodicity $\left(\bmod x^{k}\right)$ of $f_{s}$ it follows that

$$
B_{i}=(-1)^{r} \sum_{\operatorname{deg} m_{1}<k} f_{s}\left(m_{1}\right) \sum_{\substack{\text { deg } m<k \\ m=m_{1}\left(\bmod x^{k}\right)}} \frac{G_{a^{r-1-i}}^{\prime}(m)}{g_{a^{r}-1-i}} .
$$

Now for each $m_{1} \varepsilon G F[q, x]$ with $\operatorname{deg} m_{1}<k$

$$
(-1)^{r} \sum_{\substack{\text { deg } m<r \\ m=m_{2}\left(\bmod x^{k}\right)}} \frac{G_{a^{r}-1-i}^{\prime}(m)}{g_{a^{r}-1-i}}=(-1)^{r} \sum_{\operatorname{deg} m<r} \frac{G_{q^{r}-1-i}^{\prime}(m)}{g_{a^{r}-1-i}} \chi_{k}\left(m-m_{1}\right),
$$

where $\chi_{k}$ is as in $\S 3$. For each such $m_{1}$ and for all $i \geq 0$ set

$$
D_{i}\left(m_{1}\right)=(-1)^{r} \sum_{\operatorname{deg} m<r} \frac{G_{a^{r}-1-i}^{\prime}(m)}{g_{a^{r}-1-i}} C_{k}^{p^{s}}\left(m-m_{1}\right),
$$

where $C_{k}(t)$ is defined by (3.1) and $i<q^{r}$. Then by (3.4), (4.6), (4.8) and (4.9)

$$
\left|B_{i}-\sum_{\operatorname{deg} m_{1}<k} f_{s}\left(m_{1}\right) D_{i}\left(m_{1}\right)\right| \leq b^{p^{*}} \leq b^{*} .
$$

But for each $m_{1}, \operatorname{deg} C_{k}^{p^{*}}\left(t-m_{1}\right)=p^{s}\left(q^{k}-1\right)$ and so by (4.9) and the remarks following (2.13), $D_{i}\left(m_{1}\right)=0$ if $i>p^{s}\left(q^{k}-1\right)$. It follows that for such $i,\left|B_{i}\right| \leq b^{s}$ which, along with (4.5), implies that $\left|A_{i}\right| \leq b^{s}$.

It remains to be shown that (4.2) actually converges to the function $f$. As the uniform limit of (continuous) polynomial functions (4.2) represents a continuous function on $V$. Since $G F[q, x]$ is dense in $V$, it suffices to show that

$$
f\left(m^{*}\right)=\sum_{i=0}^{\infty} A_{i} \frac{G_{i}\left(m^{*}\right)}{q_{i}}
$$

for all $m^{*} \varepsilon G F[q, x]$. Suppose that $\operatorname{deg} m^{*}<d$. Then by (2.3) and (2.8) $G_{i}\left(m^{*}\right)=0$ for $i \geq q^{d}$, and so the series in (4.11) is actually finite. Let $f_{d}(t)$ be the unique polynomial over $V$ of degree $<q^{d}$ such that $f_{d}(m)=f(m)$ for all $m \varepsilon G F[q, x]$ of degree $<d$. Then application of (2.12) and (2.13) to $f_{d}(t)$ yields (4.11). The polynomials $f_{d}(t)$ also yield a simple proof of the uniqueness of the coefficients $A_{i}$ in $(4.2)[5 ; 404]$.

5. Differentiability. The following propositions will be used to discuss differentiability criteria for continuous functions on $V$.

Proposition 1. For all nonnegative integers $j$ and $k$

$$
\left(\begin{array}{c}
j+k \\
j
\end{array}\right) g_{i+k}=\left(\begin{array}{c}
j+k \\
j
\end{array}\right) g_{i} g_{k}
$$

where $g_{i}$ is defined by (2.11). 
Proof. Let $j=j_{0}+j_{1} q+\cdots+j_{s} q^{s}$ and let $k=k_{0}+k_{1} q+\cdots+k_{s} q^{s}$, where $0 \leq j_{i}, k_{i}<q$. If $j_{i}+k_{i}<q$ for each $i, 1 \leq i \leq s$, then $g_{i+k}=g_{i} g_{k}$ by (2.11). If $j_{i}+k_{i} \geq q$ for some $i$, let $n$ be the smallest such $i$. Then $j_{n}+k_{n}=$ $q+r$, where $0 \leq r<q$ and $r<j_{n}$. Then by a familiar congruence for binomial coefficients $\left(\begin{array}{c}j+k \\ j\end{array}\right)$ is congruent $(\bmod p)$ to a product of binomial coefficients, one of which is $\left(\begin{array}{l}r \\ j_{n}\end{array}\right)=0$. Hence in this case (5.1) reduces to the identity $0=0$.

Proposition 2. For all $n \geq 1$

$$
\frac{G_{n}(t)}{t g_{n-1}}=\frac{G_{q}^{\prime} \cdot(n)-1}{g_{a^{e}(n)-1}}(t) \frac{G_{n-q^{e}(n)}(t)}{g_{n-q^{e}(n)}}
$$

where $q^{e(n)} \mid n$ and $q^{e(n)+1} \nmid n$.

Proof. Let $n=n_{0}+n_{1} q+\cdots+n_{s} q^{s}$, where $0 \leq n_{i}<q$. If $n_{0}>0$, then $e(n)=0$, and so by (2.8), (2.11) and the fact that $\Psi_{0}(t)=t$

$$
\frac{G_{n}(t)}{t g_{n-1}}=\frac{\Psi_{n}^{n_{0}-1}(t) \Psi_{1}^{n_{1}}(t) \cdots \Psi_{s}^{n_{*}}(t)}{g_{n-1}}=\frac{G_{n-1}(t)}{g_{n-1}} .
$$

If $n_{0}=0$, let $j=e(n)$ be the first nonzero coefficient in the $q$-adic expansion of $n$. Then $n-1=(q-1)+(q-1) q+\cdots+(q-1) q^{i-1}+\left(n_{j}-1\right) q^{j}+$ $n_{i+1} q^{i+1}+\cdots+n_{s} q^{s}$ and $n-q^{i}=\left(n_{j}-1\right) q^{i}+n_{i+1} q^{i+1}+\cdots+n_{s} q^{8}$ so that

$$
\begin{aligned}
\frac{G_{n}(t)}{t g_{n-1}} & =\frac{\Psi_{i}(t)}{t F_{1}^{q-1} \cdots F_{i-1}^{q-1}} \frac{\Psi_{i}^{n_{j}-1}(t) \Psi_{i+1}^{n_{j+1}}(t) \cdots \Psi_{s}^{n_{s}}(t)}{F_{i}^{n_{j-1}} F_{i+1}^{n_{j+1}} \cdots F_{s}^{n_{s}}} \\
& =\frac{G_{a^{i}-1}^{\prime}(t)}{g_{a^{i}-1}} \frac{G_{n-q^{i}}(t)}{g_{n-q^{i}}}
\end{aligned}
$$

since $\Psi_{i}(t) / t=G_{a^{i}-1}^{\prime}(t)[2 ; 141]$. It follows from (5.2) that $G_{n}(t) / g_{n-1}$ is an integral valued polynomial over $G F(q, x)$ and, since $G F[q, x]$ is dense in $V$, that

$$
\left|\frac{G_{n}(t)}{t g_{n-1}}\right|_{t=\alpha} \leq 1
$$

if $|\alpha| \leq 1$

Proposition 3. For all $n \geq 1$

$$
\left(\frac{G_{n}(t)}{t g_{n-1}}\right)_{t=0}= \begin{cases}(-1)^{k} & \text { if } n=q^{k} \\ 0 & \text { otherwise. }\end{cases}
$$

Proof. This follows from (5.2), the fact that $G_{i}(0)=0$ for $i>0$ and the fact that $G_{a^{k}-1}^{\prime}(0) / g_{a^{k}-1}=(-1)^{k}[6 ; \S 5]$.

Proposition 4. For all $n \geq 1$ 


$$
\frac{g_{n-1}}{g_{n}}=\frac{1}{L_{e(n)}},
$$

where $L_{i}$ is defined by (2.6) and $e(n)$ is as in (5.2).

Proof. This follows immediately from (2.6) and (2.11).

We may now give a sufficient condition for the differentiability of a continuous function $f: V \rightarrow V$ at $u \varepsilon V$.

THEOREM C. Let $f: V \rightarrow V$ continuously and suppose that

$$
f(t)=\sum_{i=0}^{\infty} A_{i} \frac{G_{i}(t)}{g_{i}}
$$

is the interpolation series for $f$ constructed from the Carlitz polynomials. For all $u \varepsilon V$ set

$$
A_{i}(u)=\sum_{k=0}^{\infty}\left(\begin{array}{c}
j+k \\
j
\end{array}\right) A_{i+k} \frac{G_{k}(u)}{g_{k}} .
$$

If $\lim _{i \rightarrow \infty} A_{i}(u) / L_{\bullet(i)}=0$, then $f$ is differentiable at $u$ and

$$
f^{\prime}(u)=\sum_{n=0}^{\infty}(-1)^{n} \frac{A_{a^{n}}(u)}{L_{n}} .
$$

Proof. By (5.8), [1; 488, (2.3)] and Proposition 1

$$
\begin{aligned}
f(t+u) & =\sum_{i=0}^{\infty} A_{i} \frac{G_{i}^{(}(t+u)}{g_{i}}=\sum_{i=0}^{\infty} \frac{A_{i}}{g_{i}} \sum_{j=0}^{i}\left(\begin{array}{l}
i \\
j
\end{array}\right) G_{i}(t) G_{i-j}(u) \\
& =\sum_{i=0}^{\infty} \sum_{i=0}^{i}\left(\begin{array}{l}
i \\
j
\end{array}\right) A_{i} \frac{G_{i}(t)}{g_{i}} \frac{G_{i-j}(u)}{g_{i-j}}
\end{aligned}
$$

for all $t, u \varepsilon V$. Since $\left(A_{i}\right)$ is a null sequence, we may reverse the order of summation in the last series in (5.11). This yields

$$
f(t+u)=\sum_{i=0}^{\infty} A_{i}(u) \frac{G_{i}(t)}{g_{i}},
$$

where

$$
A_{i}(u)=\sum_{k=0}^{\infty}\left(\begin{array}{c}
j+k \\
j
\end{array}\right) A_{i+k} \frac{G_{k}(u)}{g_{k}} .
$$

Note that $\left(A_{i}(u)\right)$ is a null sequence and that $A_{0}(u)=f(u)$; so for all nonzero $t \varepsilon V$

$$
\frac{f(t+u)-f(u)}{t}=\sum_{j=1}^{\infty} A_{i}(u) \frac{G_{i}(t)}{t g_{i}}=\sum_{i=1}^{\infty} \frac{A_{j}(u)}{L_{e(i)}} \frac{G_{i}(t)}{t g_{i-1}}
$$

by Proposition 3. 
Now if $\left(A_{i}(u) / L_{o(j)}\right)$ is a null sequence, then by (5.5) the rightmost series in (5.14) converges for all $t \varepsilon V$ (including zero) to a continuous function on $V$. Hence $f^{\prime}(u)$ exists and by Proposition 3

$$
f^{\prime}(u)=\sum_{j=1}^{\infty}\left(\frac{A_{j}(u)}{L_{e(j)}} \frac{G_{i}(t)}{t g_{i-1}}\right)_{t=0}=\sum_{n=0}^{\infty}(-1)^{n} \frac{A_{a^{n}}(u)}{L_{n}} .
$$

We remark that the function $f$ of (5.8) is a linear operator on the $G F(q)$-vector space $V$ precisely when $A_{i}=0$ for $i$ not a power of $q[5 ; 406]$. Hence if $f$ is linear, then

$$
A_{j}(u)=\sum_{k=0}^{\infty}\left(\begin{array}{c}
j+k \\
j
\end{array}\right) A_{i+k} \frac{G_{k}(u)}{g_{k}}=A_{j}
$$

so that the condition $\lim _{i \rightarrow \infty} A_{j}(u) / L_{e(i)}=0$ is equivalent to $\lim _{n \rightarrow \infty} A_{q^{n}} / L_{n}=0$. This latter condition is, in the linear case, both necessary and sufficient for $f$ to be everywhere differentiable on $V[6 ; \S 5]$.

\section{REFERENCES}

1. L. CARlitz, $A$ set of polynomials, Duke Math. J., vol. 6(1940), pp. 486-504.

2. L. CARLITZ, On certain functions connected with polynomials in a Galois field, Duke Math. J., vol. 1(1935), pp. 137-168.

3. J. Dieudonne, Sur les fonctions continues p-adiques, Bull. Sci. Math. (2), vol. 68(1944), pp. 79-95.

4. K. MAHLER, An interpolation series for continuous functions of a p-adic variable, J. Reine Angew. Math., vol. 199(1958), pp. 23-34.

5. C. WAGNER, Interpolation series for continuous functions on $\pi$-adic completions of $G F(q, x)$, Acta Arith., vol. 17(1971), pp. 389-406.

6. C. WAGNeR, Linear operators in local fields of prime characteristic, J. Reine Agnew. Math., vol. 251(1971), pp. 153-160.

Department of Mathematics, University of Tennessee, Knoxville, Tennessee 37916 